\title{
On Solutions for a Generalized Differential Equation Arising in Boundary Layer Problem
}

\author{
Sergey Smirnov \\ Institute of Mathematics and Computer Science, University of Latvia, Raina Bulvaris 29, Riga, LV-1459, Latvia \\ Correspondence should be addressed to Sergey Smirnov; srgsm@inbox.lv
}

Received 29 November 2013; Revised 14 February 2014; Accepted 14 February 2014; Published 18 March 2014

Academic Editor: Sivaguru Sritharan

Copyright (C) 2014 Sergey Smirnov. This is an open access article distributed under the Creative Commons Attribution License, which permits unrestricted use, distribution, and reproduction in any medium, provided the original work is properly cited.

We treat the existence and uniqueness of a solution for the generalized Blasius problem which arises in boundary layer theory. The shooting method is used in the proof of our main result. An example is included to illustrate the results.

\section{Introduction}

The steady motion in the boundary layer along a thin flat plate which is immersed at zero incidence in a uniform stream with constant velocity can be described [1] in terms of the solution of the differential equation:

$$
x^{\prime \prime \prime}=-x x^{\prime \prime}
$$

which satisfies the boundary conditions

$$
x(0)=x^{\prime}(0)=0, \quad x^{\prime}(\infty)=1 .
$$

This problem was first solved numerically by Blasius [2] and is the subject of a vast literature.

Some generalizations of the Blasius equation can be found in [3-5]. In [3], the authors investigate the model $\left(\left|x^{\prime \prime}\right|^{n-1} x^{\prime \prime}\right)^{\prime}+(1 /(n+1)) x x^{\prime \prime}=0, x(0)=x^{\prime}(0)=0, x^{\prime}(\infty)=$ 1 , arising in the study of a laminar boundary layer for a class of non-Newtonian fluids. In [4], the author considers the equation $\left[a(t) x^{\prime \prime}\right]^{\prime}+x x^{\prime \prime}=0$, which describes boundary layer flows with temperature dependent viscosity.

It is our goal to study the existence of solutions to the generalized boundary value problem consisting of the nonlinear third order differential equation

$$
x^{\prime \prime \prime}=-a(t) f(x) x^{\prime \prime}
$$

subject to the boundary conditions (2). We assume that the functions $a: \mathbb{R} \rightarrow[0, \infty), a(t) \neq \equiv \quad 0$, and $f: \mathbb{R} \rightarrow \mathbb{R}$ are continuous. The additional conditions imposed on $a$ and $f$ in (3) are the following ones:

(H1) $a(B t)=B^{k} a(t)$ for some $k \geq 0$ and all $B>0$;

(H2) $x f(x)>0$ for $x \neq 0$;

(H3) $f(B x)=B^{q} f(x)$ for some $q \geq 1$ and all $B>0$.

An example of (3), which satisfies the conditions (H1), (H2), and (H3), is $x^{\prime \prime \prime}=-t^{2} x^{3} x^{\prime \prime}$. If $k=0$ and $q=1$, then (3) coincides with the Blasius equation (1).

For the related Falkner-Skan equation [6] $x^{\prime \prime \prime}+x x^{\prime \prime}+\beta(1-$ $\left.\left(x^{\prime}\right)^{2}\right)=0$ the similar generalization was given in [7]. FalknerSkan equation describes the steady two-dimensional flow of a slightly viscous incompressible fluid past a wedge of angle $\pi \beta(0 \leq \beta<2)$.

The shooting method [8] is used for treating the existence and the number of solutions to boundary value problem. The shooting method reduces solving a boundary value problem to solving of an initial value problem. So we consider solution $x(t, \gamma)$ of the auxiliary initial value problem for (3) with initial data

$$
x(0)=x^{\prime}(0)=0, \quad x^{\prime \prime}(0)=\gamma
$$

and we are looking for $\gamma_{1}$ and $\gamma_{2}$ such that $x^{\prime}\left(\infty, \gamma_{1}\right)>1$ and $x^{\prime}\left(\infty, \gamma_{2}\right)<1$. Applying the intermediate value theorem, continuity of $x(t, \gamma)$ with respect to $\gamma$ leads to the existence of at least one $\gamma^{*}$ such that $x^{\prime}\left(\infty, \gamma^{*}\right)=1$.

The paper is organized as follows. Section 2 contains some auxiliary results. Section 3 is devoted to the properties of 
solutions of initial value problem (3), (4). In Section 4 we consider dependence of solutions on initial data. In Section 5 we deal with solutions to boundary value problem (3), (2). Also one example is given to illustrate the results. The ideas of the proofs of some results are taken from [6].

\section{Preliminary Results}

Proposition 1. Suppose that a function a $(t)$ satisfies assumption (H1). If $k>0$, then $a(t)$ is increasing for $t>0$ and decreasing for $t<0$. If $k=0$, then $a(t)=$ Const $>0$.

Proof. Assume $k>0$. First, let us prove that the function $a(t)$ is increasing for $t>0$. Let us choose $0<t_{1}<t_{2}$. Obviously, there exists a $B>1$ such that $t_{2}=B t_{1}$. Now consider $a\left(t_{2}\right)=$ $a\left(B t_{1}\right)=B^{k} a\left(t_{1}\right) \geq a\left(t_{1}\right)$ (we use the assumption $a(t) \geq 0$ to obtain the last inequality). Thus, $a(t)$ is an increasing function for $t>0$. Next, let us prove that the function $a(t)$ is decreasing for $t<0$. Let us choose $t_{2}<t_{1}<0$. Obviously, there exists a $B>1$ such that $t_{2}=B t_{1}$. Now consider $a\left(t_{2}\right)=a\left(B t_{1}\right)=$ $B^{k} a\left(t_{1}\right) \geq a\left(t_{1}\right)$. Thus, $a(t)$ is a decreasing function for $t<0$.

Assume $k=0$. It follows that $a(t)=$ Const $>0$, since for any $B>0 a(B t)=B^{0} a(t)=a(t)$.

Proposition 2. If a function $f(x)$ satisfies assumptions (H2) and (H3), then $f(x)$ is strictly increasing.

Proof. We can obtain that the function $f(x)$ is strictly increasing repeating the arguments used in the proof of the previous proposition.

\section{Properties of Solutions}

Proposition 3. Let $x(t, \gamma)$ be a solution of initial value problem (3), (4). Let $\left[0, t_{\gamma}\right), t_{\gamma} \leq \infty$, be the interval of existence of $x(t, \gamma)$. If $\gamma \neq 0$, then $x^{\prime \prime}(t, \gamma) \neq 0$ for $0<t<t_{\gamma}$.

Proof. Without loss of generality, let $x^{\prime \prime}(0)>0$; then $x^{\prime \prime}(t, \gamma)$ will be positive in some open interval with the left boundary point $t=0$. Suppose that there exists a point $t=t_{0}>0$ such that $x^{\prime \prime}\left(t_{0}\right)=0$ and $x^{\prime \prime}(t)>0$ for $0<t<t_{0}<t_{\gamma}$. Dividing (3) by $x^{\prime \prime}$ and integrating from 0 to $t_{0}$, we obtain

$$
x^{\prime \prime}\left(t_{0}\right)=x^{\prime \prime}(0) e^{-\int_{0}^{t_{0}} a(s) f(x(s)) d s} .
$$

The right side is positive and $x^{\prime \prime}\left(t_{0}\right)>0$. This contradiction proves the proposition.

Corollary 4. Let $x(t, \gamma)$ be a solution of initial value problem (3), (4). If $\gamma>0$, then $x^{\prime}(t, \gamma)$ and $x(t, \gamma)$ are positive and increasing functions for $0<t<t_{\gamma}$. Moreover if condition (H2) is satisfied, then $x^{\prime \prime}(t, \gamma)$ is a decreasing function for $0<t<t_{\gamma}$. If $\gamma<0$, then $x^{\prime}(t, \gamma)$ and $x(t, \gamma)$ are negative and decreasing functions for $0<t<t_{\gamma}$.

Proof. Let $\gamma>0$. By Proposition $3 x^{\prime \prime}(t, \gamma)>0$ and therefore $x^{\prime}(t, \gamma)$ is increasing and, in view of $x^{\prime}(0)=0, x^{\prime}(t, \gamma)>0$ for $0<t<t_{\gamma}$. Since $x^{\prime}(t, \gamma)>0$, then $x(t, \gamma)$ is increasing and, in view of $x(0)=0, x(t, \gamma)>0$ for $0<t<t_{\gamma}$.

Let (H2) hold. Since $x(t, \gamma)>0, x^{\prime}(t, \gamma)>0$, then in view of (3) $x^{\prime \prime \prime}(t, \gamma)<0$ and $x^{\prime \prime}(t, \gamma)$ is a decreasing function for $0<t<t_{\gamma}$.

For $\gamma<0$ the proof is analogous.

Proposition 5. Let $x(t)$ be a solution of (3). If one of the functions $x(t), x^{\prime}(t)$, or $x^{\prime \prime}(t)$ tends to infinity as $t \rightarrow t^{*}$ then the others also tend to infinity.

Proof. If $x^{\prime \prime}(t)$ is bounded for $t \rightarrow t^{*}$ then $x^{\prime}(t)$ and $x(t)$ also are bounded. If $x^{\prime}(t)$ is bounded for $t \rightarrow t^{*}$ then $x(t)$ also is bounded and, by formula (5), $x^{\prime \prime}(t)$ is bounded. If $x(t)$ is bounded then, by formula $(5), x^{\prime \prime}(t)$ is bounded and $x^{\prime}(t)$ also is bounded.

Proposition 6. Let $x(t, \gamma)$ be a solution of initial value problem (3), (4). If $\gamma>0$ then $x(t, \gamma)$ is defined for all $t \geq 0$ $\left(t_{\gamma}=\infty\right)$.

Proof. If $x(t, \gamma)$ is defined only in a finite interval $\left[0, t^{*}\right)$ then, by Proposition $5, x(t), x^{\prime}(t)$, and $x^{\prime \prime}(t)$ tend to $+\infty$ as $t \rightarrow$ $t^{*}$. But $x^{\prime \prime}(t)$ is a positive decreasing function and therefore bounded near $t^{*}$. This contradiction proves the proposition.

Proposition 7. Let $x(t, \gamma)$ be a solution of initial value problem (3), (4). If $\gamma>0$ and conditions (H1), (H2), and (H3) hold, then there exists a positive constant $\lambda$ such that $\lim _{t \rightarrow+\infty} x^{\prime}(t, \gamma)=$ $\lambda$.

Proof. Since $x(t, \gamma)$ and $x^{\prime}(t, \gamma)$ are positive and increasing functions (Corollary 4), then there exists $t_{1}>0$ such that for $t>t_{1} x^{\prime}(t, \gamma) \geq C_{1}>0$ and $x(t, \gamma) \geq C_{1} t$. In view of $a(t)>0$ is increasing for $t>0$, it follows that there exists $t_{0}>t_{1}$ such that for $t>t_{0} a(t) f(x(t))=a(t) x(t)^{q} f(1)>1$. Thus, $x^{\prime \prime \prime}<-x^{\prime \prime}$ for $t>t_{0}$. Dividing the last inequality by $x^{\prime \prime}$ and integrating from $t_{0}$ to $t$, we obtain

$$
0<x^{\prime \prime}(t)<x^{\prime \prime}\left(t_{0}\right) e^{-\left(t-t_{0}\right)} .
$$

Since $\int_{t_{0}}^{+\infty} x^{\prime \prime}\left(t_{0}\right) e^{-\left(s-t_{0}\right)} d s=x^{\prime \prime}\left(t_{0}\right)$ converges, then (by comparison test) $\int_{t_{0}}^{+\infty} x^{\prime \prime}(s) d s=\lim _{t \rightarrow+\infty} \int_{t_{0}}^{t} x^{\prime \prime}(s) d s=$ const $>0$ or $\lim _{t \rightarrow+\infty} x^{\prime}(t)-x^{\prime}\left(t_{0}\right)=$ const $>0$. Therefore, there exists a positive constant $\lambda$ such that $\lim _{t \rightarrow+\infty} x^{\prime}(t, \gamma)=$ $\lambda$.

\section{Scaling Formula}

Proposition 8. Suppose that conditions (H1) and (H3) are fulfilled. If $x(t)$ is a solution of (3), then the function

$$
y(t)=B^{((1+k) / q)} x(B t),
$$

where $B>0$ is an arbitrary constant, is also a solution of (3). 
Proof. The proposition can be proved by direct substitution. So,

$$
\begin{aligned}
y^{\prime \prime \prime}(t) & =B^{((1+k) / q)+3} x^{\prime \prime \prime}(B t), \\
f(y(t)) & =B^{((1+k) / q) q} f(x(B t)), \\
y^{\prime \prime}(t) & =B^{((1+k) / q)+2} x^{\prime \prime}(B t) .
\end{aligned}
$$

Then,

$$
\begin{aligned}
B^{((1+k) / q)+3} x^{\prime \prime \prime}(B t) \\
=-a(t) B^{((1+k) / q) q} f(x(B t)) B^{((1+k) / q)+2} x^{\prime \prime}(B t) \\
=-a(B t) B^{-k} B^{((1+k) / q) q} f(x(B t)) B^{((1+k) / q)+2} x^{\prime \prime}(B t) \\
=B^{-k} B^{((1+k) / q) q} B^{((1+k) / q)+2} x^{\prime \prime \prime}(B t) .
\end{aligned}
$$

Therefore,

$$
\begin{aligned}
& B^{((1+k) / q)+3}=B^{-k} B^{((1+k) / q) q} B^{((1+k) / q)+2}, \\
& \text { or } \quad B^{((3 q+k+1) / q)}=B^{((3 q+k+1) / q) .}
\end{aligned}
$$

Hence the proof.

Proposition 9. Suppose that conditions (H1) and (H3) are fulfilled. If $x\left(t, \gamma_{0}\right)$ is a solution of (3) such that

$$
x(0)=x^{\prime}(0)=0, \quad x^{\prime \prime}(0)=\gamma_{0} \neq 0,
$$

then every solution of (3) which has a double zero at $t=0$ and the second derivative $\gamma$ at $t=0$ of the same sign as $\gamma_{0}\left(\gamma \gamma_{0}>0\right)$ can be expressed via solution $x\left(t, \gamma_{0}\right)$ as

$$
y(t, \gamma)=\left(\frac{\gamma}{\gamma_{0}}\right)^{(1+k) /(1+k+2 q)} x\left(\left(\frac{\gamma}{\gamma_{0}}\right)^{q /(1+k+2 q)} t, \gamma_{0}\right) .
$$

Proof. The proof follows from Proposition 8 and direct substitution. So,

$$
y^{\prime \prime}(0)=B^{((1+k) / q)+2} \gamma_{0}=\gamma \quad \text { or } \quad B=\left(\frac{\gamma}{\gamma_{0}}\right)^{(q /(1+k+2 q))} .
$$

The proof is complete.

\section{Existence of Solutions for Boundary Value Problem}

Theorem 10. Suppose that conditions (H1), (H2), and (H3) are fulfilled, then boundary value problem (3), (2) has a unique solution.

Proof. Consider the solution $x(t, \gamma)$ of auxiliary initial value problem (3), (4). By Proposition 7 there exists a positive constant $\lambda$ such that $\lim _{t \rightarrow+\infty} x^{\prime}\left(t, \gamma_{0}\right)=\lambda$. By Proposition 9 the function defined by formula (12) is also a solution of auxiliary initial value problem (3), (4) and

$$
y^{\prime}(t, \gamma)=\left(\frac{\gamma}{\gamma_{0}}\right)^{(1+k+q) /(1+k+2 q)} x^{\prime}\left(\left(\frac{\gamma}{\gamma_{0}}\right)^{q /(1+k+2 q)} t, \gamma_{0}\right) .
$$

Obviously, there exists a unique $\gamma=\gamma^{*}>0$ such that $\left(\gamma^{*} / \gamma_{0}\right)^{(1+k+q) /(1+k+2 q)}=1 / \lambda$. Hence the proof.



FIGURE 1: Solution and its derivatives of problem (15).

Example 11. Consider the problem

$$
\begin{gathered}
x^{\prime \prime \prime}=-t^{2} x^{3} x^{\prime \prime}, \\
x(0)=x^{\prime}(0)=0, \quad x^{\prime}(\infty)=1 .
\end{gathered}
$$

The nonlinearities in the equation are chosen for the convenience of construction of the example. Conditions ( $\mathrm{H1}$ ), $(\mathrm{H} 2)$, and (H3) are fulfilled; then boundary value problem (15) has a unique solution. Solution $x(t)$ and its derivatives $x^{\prime}(t)$ and $x^{\prime \prime}(t)$ of boundary value problem (15) are presented in Figure 1, which illustrates the main theorem. There is a solution $x(t)$ such that $x(0)=0, x^{\prime}(0)=0$, and $x^{\prime}(\infty)=1$. The derivative $x^{\prime}(t) \rightarrow 1$ as $t \rightarrow+\infty$ and $x^{\prime \prime}(t) \rightarrow 0$ as $t \rightarrow+\infty$. The Wolfram Mathematica 7.0 package was used to construct the graphs.

\section{Conflict of Interests}

The author declares that there is no conflict of interests regarding the publication of this paper.

\section{Acknowledgment}

This research has been supported by the European Social Fund within Project no. 2013/0024/1DP/1.1.1.2.0/13/APIA/ VIAA/045.

\section{References}

[1] H. Schlichting and K. Gersten, Boundary-Layer Theory, Springer, Berlin, Germany, 2000.

[2] H. Blasius, "Grenzschichten in Flussigkeiten mit kleiner Reibung," Zeitschrift für angewandte Mathematik und Physik, vol. 56, pp. 1-37, 1908.

[3] M. Benlahsen, M. Guedda, and R. Kersner, "The generalized Blasius equation revisited," Mathematical and Computer Modelling, vol. 47, no. 9-10, pp. 1063-1076, 2008.

[4] T. Fang, "Influences of fluid property variation on the boundary layers of a stretching surface," Acta Mechanica, vol. 171, pp. 105$118,2004$.

[5] T. Fang, F. Guo, and C. F. Lee, "A note on the extended Blasius equation," Applied Mathematics Letters, vol. 19, no. 7, pp. 613-617, 2006. 
[6] W. A. Coppel, "On a differential equation of boundary-layer theory," Philosophical Transactions of the Royal Society of London. Series A, vol. 253, pp. 101-136, 1960.

[7] B. Brighi and J.-D. Hoernel, "On a general similarity boundary layer equation," Acta Mathematica Universitatis Comenianae, vol. 77, no. 1, pp. 9-22, 2008.

[8] S. P. Hastings and J. B. McLeod, Classical Methods in Ordinary Differential Equations: With Applications to Boundary Value Problems, vol. 129 of Graduate Studies in Mathematics, American Mathematical Society, Providence, RI, USA, 2012. 




Advances in

Operations Research

mansans



The Scientific World Journal
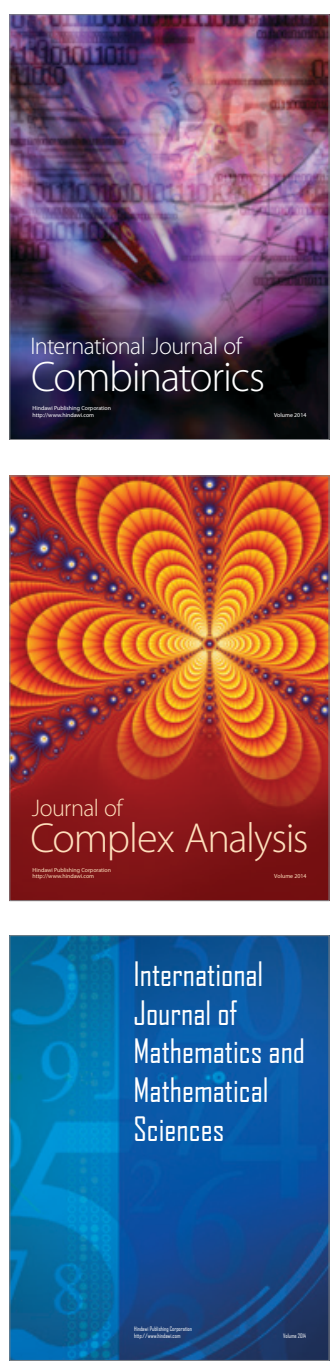


Submit your manuscripts at http://www.hindawi.com
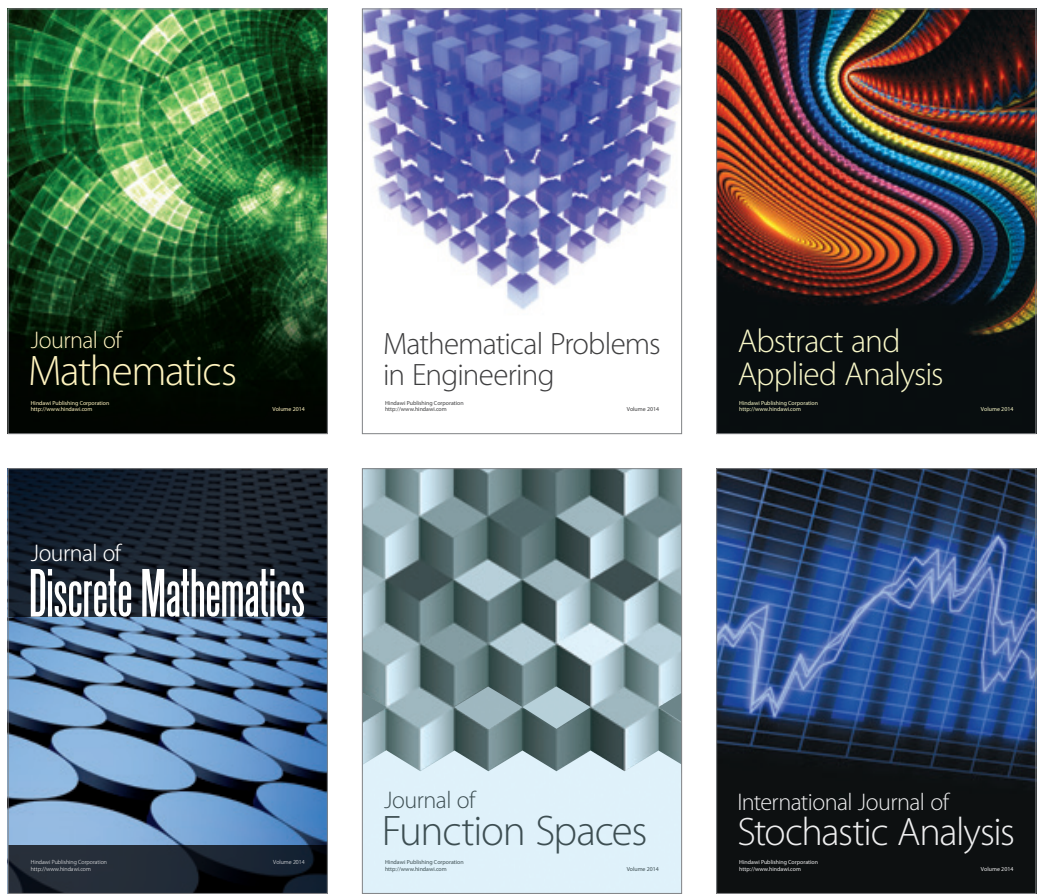

Journal of

Function Spaces

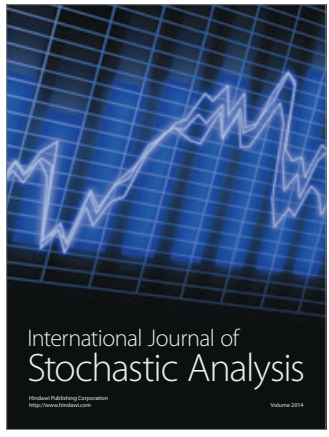


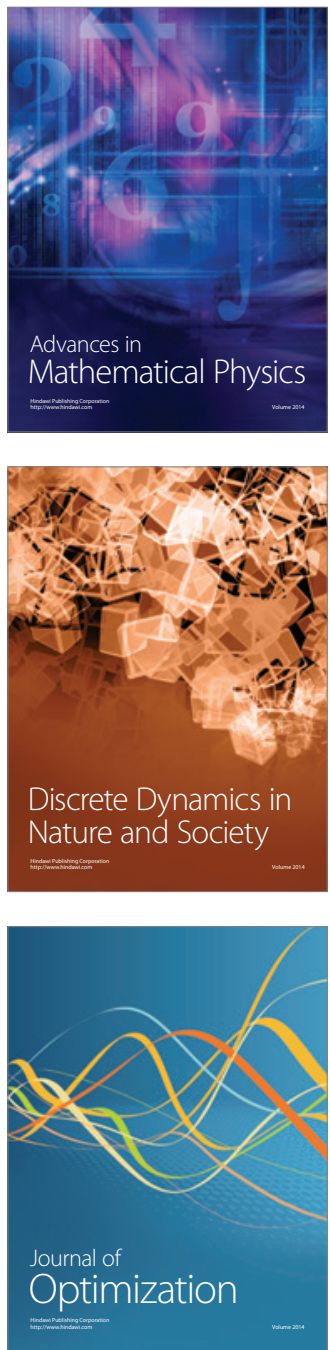